\section{Orientation of Protein Determined in QD-Bioconjugate Assembly}

One class of hybrid nanomaterials that bridges the interface between materials science and biology is the protein-nanomaterial composite. Beneficial protein properties imparted by design to such nanocomposites include self-assembly assistance, biorecognition, and catalysis. The interaction between protein bioreceptors and the inorganic nanocomponents is fundamentally important. Recently, quantum dots (QDs) were bound to maltose-binding proteins (MBPs) to make solution-phase biosensors that function by changes in fluorescence resonance energy transfer (FRET). Although the biosensors' activities were evident, the nature of the MBP-QD interaction was unknown. In the work discussed here, I.L. Medintz, J.H. Konnert, and co-workers at the Naval Research Laboratory in Washington, D.C., have developed a FRET-based modeling technique to determine the orientation of MBP coordinated to the QD surface.

As reported in the June 29 issue of the Proceedings of the National Academy of Sciences (p. 9612), the researchers prepared MBP-QD assemblies from CdSe-ZnS core-shell QDs and six different MBP mutants in which single cysteine substitutions were spatially distributed on the protein surface and labeled with rhodamine red (RR) dye. The core-shell QDs were made water soluble by using bidentate dihydrolipoic acid to replace the organic capping shell left over from the colloidal synthesis process. MBP, an ellipsoidal protein with dimensions of about $30 \AA \times 40 \AA \times 65 \AA$, coordinates to the Zn-S surface of each spherical QD (core-shell diameter, $\sim 60 \AA$ ) by a C-terminal oligohistidine segment. The researchers determined from FRET efficiency data the distances, $d_{i}$, from the energy-donating QD to the six different RR-acceptor locations. These distances were used in conjunction with the MBP crystal structure to model the orientation of MBP with respect to the QD surface using a method the researchers liken to a nanoscale global positioning system, that is, triangulating a point on the globe from orbiting satellites. The protein's dye-acceptor positions relative to a spherical QD are analogous to satellite positions about the earth. The researchers said that this approach should, in principle, allow determination of the QD orientation with respect to MBP with as few as four non-coplanar locations instead of the 21 parameters that define the six MBP-dye acceptor positions and the QD center. The researchers, however, resorted to an iterative method that minimized the mean-square deviations of the
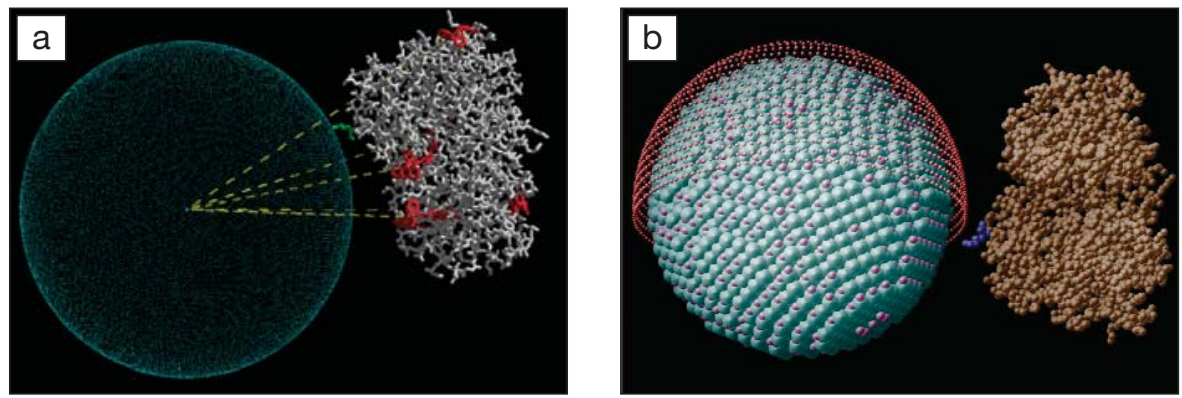

Figure 1. Refined maltose-binding protein (MBP) orientation relative to a quantum dot (QD). (a) Side view with all six rhodamine red structures positioned (in red). The refined distances to the center of the $Q D$ are shown in yellow. The distance from the nearest MBP atom is shown in green. (b) View of the final refined orientation of $M B P$ relative to the $Q D$, with $Q D$ surface sulfur atoms in teal and zinc atoms in pink. The red shell shows the estimated outer radius of the dihydrolipoic acid ligand. Reproduced with permission from PNAS; () National Academy of Sciences, USA 2004.

measured and computed $d_{i}$ values.

The research team found that MBP has a preferred orientation on the QD surface (see Figure 1), although it appears that MBP is free to rotate about the pentahistidine that attaches to the QD. Medintz, Konnert, and their colleagues believe that their approach can be used to determine the orientation of other proteins on a QD or any other spherical nanoparticle and, indeed, may be extended to other labeled biomaterials attached to any nanoparticle surface.

SteVen TrohalaKi

\section{Surface Plasmons Utilized to Achieve High-Density Nanolithography}

The semiconductor industry is under constant pressure to reduce feature size in the photolithographic processing of integrated circuits in order to increase transistor density and computing power; however, using new lithography techniques that overcome the diffraction limit of the light are still costly. W. Srituravanich, $X$. Zhang, and colleagues at the University of California, Los Angeles, have developed an economically viable process called plasmonic nanolithography (PNL) that utilizes surface plasmons transmitted through subwavelength two-dimensional holearray masks.

The researchers reported in the June 9 issue of Nano Letters (p. 1085) the fabrication of $90 \mathrm{~nm}$ dot-array patterns on a $170 \mathrm{~nm}$ period using PNL, which is well below the diffraction limit of far-field optical lithography. They observed strong UV light transmission and wavelength-dependent transmission in their far-field transmission measurements, confirming surface plasmons as active contributors in the lithography process.
The researchers designed a plasmonic $\mathrm{Al}$ mask perforated with $2 \mathrm{D}$ periodic hole arrays surrounded by two dielectric layers with quartz as the supporting substrate and a UV-transparent poly(methyl methacrylate) (PMMA) spacer layer (30-80 nm thick) with comparable dielectric constants of 2.18 and 2.30 at the exposure wavelength of $365 \mathrm{~nm}$. They fabricated 40-nm-wide hole-array masks with $170 \mathrm{~nm}, 220 \mathrm{~nm}$, or $250 \mathrm{~nm}$ periods on 80-nm-thick Al films using focused ionbeam (FIB) milling, with the $220 \mathrm{~nm}$ period corresponding to $(1,0)$ and $(0,1)$ resonance modes.

After FIB milling, a 30-nm-thick spacer layer of PMMA was spun on, followed by a layer of photoresist (SU-8). After development, atomic force microscopy revealed feature sizes as small as $90 \mathrm{~nm}$, which is equivalent to $\sim \lambda / 4$ of the used exposure light wavelength, and with a pattern period of $<\lambda / 2$, which is far beyond the diffraction limit of far-field lithography. The optimal exposure time was only $9 \mathrm{~s}$, corresponding to a fluence of $72 \mathrm{~mJ} / \mathrm{cm}^{2}$. This is comparable to typical exposure fluences used in conventional lithography, with large features implying strong near-field transmission enhancement by surface plasmons, which gives it great economic potential. Even smaller features can be realized by reducing the spacer layer thickness, opening up high-resolution, high-density nanolithography with high transmittance using conventional light sources, which would eliminate the need for complex vacuum setups such as are needed for extreme UV lithography.

The PNL technique may find application in biosensing, photonic crystals, and high-density magnetic storage. The plasmon concept applies not only to periodic 
structures, but also to arbitrary shapes. Currently, Zhang and colleagues are developing fully parallel arbitrary patterning plasmonic lithography.

Alfred A. ZINN

\section{Defects in Solar-Cell Materials Enhance Efficiency}

Scientists working to improve solar cells have traditionally sought to eliminate defects found in their materials. However, it has been known for some time that polycrystalline solar cells with grain-boundary defects can be more efficient than their single-crystal counterparts. I. Visoly-Fisher, D. Cahen, and $\mathrm{S}$. Cohen of the Weizmann Institute in Israel and A. Ruzin of Tel Aviv University have recently used a number of scanning probe techniques to determine how grainboundary defects improve the performance of one type of polycrystalline solar cell. As reported in the June issue of Advanced Materials (p. 879), the researchers characterized the electrical properties of single grain boundaries and grain surfaces of $\sim 1 \mu \mathrm{m}$ CdTe grains in $p$-CdTe $/ n$-CdS polycrystalline thin-film solar cells. The tools used included scanning capacitance microscopy, scanning Kelvin probe microscopy, atomic force microscopy (AFM), and conductive probe AFM.

Most commercial solar cells are made of single-crystal semiconductors such as silicon, but single crystals are expensive to manufacture. The more cost-effective solar cells made of polycrystalline films were found to outperform their single-crystal counterpart cells, which was an unexpected outcome. When light strikes the solar cell, the semiconductor within it serves as an antenna, absorbing the light energy and allowing the free flow of electrons

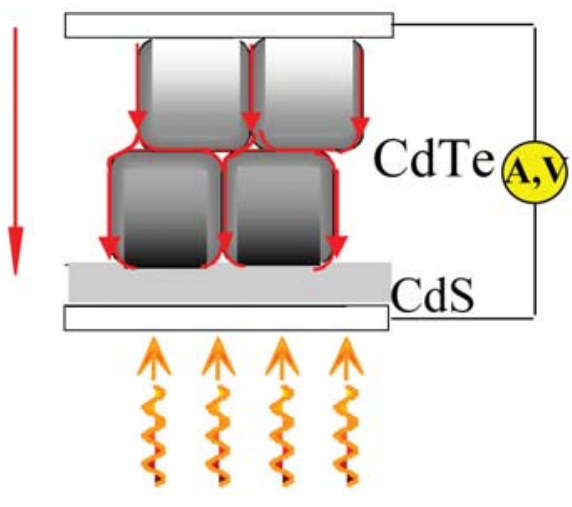

Figure 1. Grain boundaries within solarcell films can improve the light-toelectricity conversion because they provide a path along which free electrons are efficiently collected and channeled. present in the semiconductor. These electrons are then harvested as an electric current for external use. The researchers at Weizmann Institute demonstrated that grain boundaries within $\mathrm{CdTe} / \mathrm{CdS}$ polycrystalline solar cells improve the light-toelectricity conversion because they provide a path along which the free electrons are efficiently collected and channeled on their way out (see Figure 1). The researchers indicated that the efficient collection of electrons is due to the beneficial effects of the $\mathrm{CdCl}_{2}$ vapor treatment that occurs during processing of these cells. The treatment getters both defects and impurities along the grain boundaries and alters the doping profile to facilitate spatial separation of electrons and holes, thus reducing recombination.

\section{Large-Scale Synthesis of Nearly Monodisperse Au and $\mathrm{Ag}$ Nanoparticles Achieved}

Gold and silver nanoparticles are important in diverse areas ranging from fundamental research to applications in electronics (single-electron transistors), catalysis, chemical sensors, and biosensors. These applications require nanoparticles in the $2-100 \mathrm{~nm}$ range that are surfacederivatized with hydrophobic and hydrophilic surfactants. H. Hiramatsu and F.E. Osterloh of the University of California, Davis, have demonstrated an inexpensive, versatile, and reproducible method for the large-scale synthesis of organoamine-protected gold and silver nanoparticles in the 6-21 nm (Au) and 8-32 nm (Ag) range and with polydispersities as low as $6.9 \%$.

Usually such nanoparticles have been fabricated by either the citrate method or the two-phase (Brust) method. The citrate method produces nearly monodispersed gold nanoparticles in the size range of 2-100 nm; however, the resulting solutions have a low nanoparticle content, and the solvent choice is restricted to water. Moreover, the resulting nanoparticles cannot be surface-derivatized with hydrophobic surfactants. The two-phase method, on the other hand, allows the introduction of hydrophobic thiols as surfactants, but it suffers from the limitation to small particle sizes $(<10 \mathrm{~nm})$, greater polydispersity, and higher costs for the phase transfer agents.

The synthesis described by Hiramatsu and Osterloh in the June 29 issue of Chemistry of Materials (p. 2509) requires only three reagents, namely, tetrachloroauric acid or silver acetate, oleylamine, and a solvent. The particles are stable in solid form and can be easily modified with hydrophobic and hydrophilic thiols to

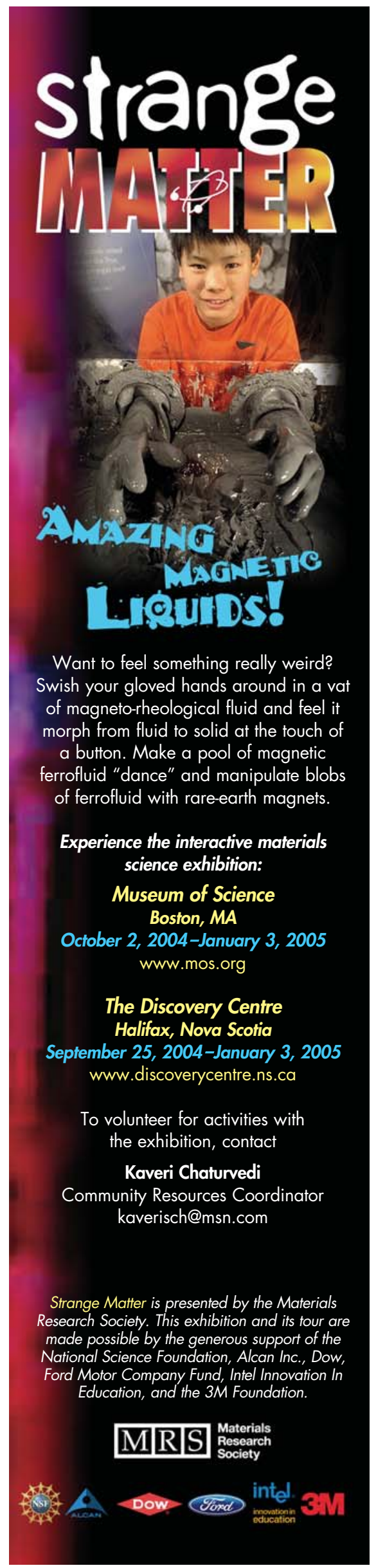

